\title{
Adolescent health in the Eastern Mediterranean Region: findings from the global burden of disease 2015 study
}

\author{
GBD 2015 Eastern Mediterranean Region Adolescent Health Collaborators ${ }^{1}$
}

Received: 1 May 2017/Revised: 15 June 2017/Accepted: 21 June 2017/Published online: 3 August 2017

(c) The Author(s) 2017. This article is an open access publication

\begin{abstract}
Objectives The 22 countries of the East Mediterranean Region (EMR) have large populations of adolescents aged 10-24 years. These adolescents are central to assuring the health, development, and peace of this region. We described their health needs.

Methods Using data from the Global Burden of Disease Study 2015 (GBD 2015), we report the leading causes of mortality and morbidity for adolescents in the EMR from 1990 to 2015 . We also report the prevalence of key health risk behaviors and determinants.

Results Communicable diseases and the health consequences of natural disasters reduced substantially between 1990 and 2015. However, these gains have largely been offset by the health impacts of war and the emergence of non-communicable diseases (including mental health disorders), unintentional injury, and self-harm. Tobacco smoking and high body mass were common health risks
\end{abstract}

This article is part of the supplement "The state of health in the Eastern Mediterranean Region, 1990-2015".

The members of GBD (Global Burden of Disease) 2015 Eastern Mediterranean Region Adolescent Health Collaborators are listed at the end of the article. Ali H. Mokdad, on behalf of GBD 2015 Eastern Mediterranean Region Adolescent Health Collaborators, is the corresponding author.

Electronic supplementary material The online version of this article (doi:10.1007/s00038-017-1003-4) contains supplementary material, which is available to authorized users.

GBD 2015 Eastern Mediterranean Region Adolescent

Health Collaborators

mokdaa@uw.edu

1 Institute for Health Metrics and Evaluation, University of Washington, Seattle, WA, USA amongst adolescents. Additionally, many EMR countries had high rates of adolescent pregnancy and unmet need for contraception.

Conclusions Even with the return of peace and security, adolescents will have a persisting poor health profile that will pose a barrier to socioeconomic growth and development of the EMR.

Keywords Adolescent health · Burden of disease $\cdot$ Eastern Mediterranean Region

\section{Introduction}

The World Health Organization's Eastern Mediterranean Region (EMR) is an administrative region of 22 countries (Table 1) that while rich in natural resources, has marked country-level variation in socioeconomic wealth (ranging from \$US 549.3 per capita in Somalia to \$US 73,653.4 per capita in Qatar), health system capacities and health coverage (Blair et al. 2014; Mandil et al. 2013; WHO 2017b). Many countries in the EMR have recently experienced social and political instabilities, civil unrest, war, and mass displacement of people (Mokdad et al. 2016a). As a result, health in many EMR countries has failed to improve in recent years (Mokdad et al. 2016a, 2014). As other papers in this series highlight, there is now an increasing burden of many preventable health problems including HIV, mental health disorders, and intentional injury (GBD 2015 Eastern Mediterranean Region HIV/AIDS Collaborators and Mokdad 2017; GBD 2015 Eastern Mediterranean Region Intentional Injuries Collaborators and Mokdad 2017; GBD 2015 Eastern Mediterranean Region Mental Health Collaborators and Mokdad 2017). There is a risk that without urgent action, the health status of this region will only 
Table 1 Eastern Mediterranean region: Countries, adolescent population and socioeconomic development, 1990-2015 (World Bank, Global Burden of Disease Study 2015, Eastern Mediterranean Countries, 1990-2015)

\begin{tabular}{|c|c|c|c|c|c|c|}
\hline \multirow[t]{2}{*}{ Country } & \multirow{2}{*}{$\begin{array}{l}\text { Proportion of country population aged } \\
10-24 \text { year in } \% \text { ( } n \text {, number of } \\
\text { adolescents in each country) }\end{array}$} & \multirow{2}{*}{$\begin{array}{l}\text { Proportion of the } \\
\text { EMR adolescent } \\
\text { population }(\%)\end{array}$} & \multirow{2}{*}{$\begin{array}{l}\text { GDP per capita } \\
\text { (\$US) }\end{array}$} & \multicolumn{3}{|c|}{ Socio-demographic index (SDI) } \\
\hline & & & & 1990 & 2015 & SDI level \\
\hline Afghanistan & $34.8 \%(11,356,556)$ & 6.2 & 594.3 & 0.1440 & 0.2888 & Low \\
\hline Bahrain & $21.7 \%(296,971)$ & 0.2 & $22,600.2$ & 0.5969 & 0.7764 & High-middle \\
\hline Djibouti & $30.5 \%(271,064)$ & 0.1 & 1945.1 & 0.3228 & 0.4615 & Low-middle \\
\hline Egypt & $26.9 \%(24,492,800)$ & 13.3 & 3614.7 & 0.4409 & 0.6191 & Middle \\
\hline Iran & $22.8 \%(17,992,150)$ & 9.8 & - & 0.4600 & 0.7154 & High-middle \\
\hline Iraq & $31.2 \%(11,348,292)$ & 6.2 & 4943.8 & 0.3997 & 0.5756 & Middle \\
\hline Jordan & $29.9 \%(2,263,213)$ & 1.2 & 4940.0 & 0.4967 & 0.6949 & High-middle \\
\hline Kuwait & $19.1 \%(745,077)$ & 0.4 & $29,300.6$ & 0.6911 & 0.8624 & High \\
\hline Lebanon & $28.5 \%(1,643,663)$ & 0.9 & 8047.6 & 0.5698 & 0.7547 & High-middle \\
\hline Libya & $25.0 \%(1,574,514)$ & 0.9 & - & 0.4747 & 0.6430 & Middle \\
\hline Morocco & $26.0 \%(8,932,361)$ & 4.8 & 2878.2 & 0.3347 & 0.4959 & Low-middle \\
\hline Oman & $21.4 \%(960,174)$ & 0.5 & $15,550.7$ & 0.4089 & 0.7301 & High-middle \\
\hline Pakistan & $30.2 \%(57,088,761)$ & 31 & 1434.7 & 0.2786 & 0.4676 & Low-middle \\
\hline Palestine & $33.6 \%(1,569,806)$ & 0.9 & - & 0.4229 & 0.5670 & Middle \\
\hline Qatar & $19.3 \%(429,261)$ & 0.2 & $73,653.4$ & 0.6162 & 0.8045 & High-middle \\
\hline Saudi Arabia & $24.4 \%(7,683,094)$ & 4.2 & $20,481.7$ & 0.5245 & 0.7593 & High-middle \\
\hline Somalia & $32.7 \%(3,545,571)$ & 1.9 & 549.3 & 0.1158 & 0.1506 & Low \\
\hline Sudan & $32.1 \%(12,950,382)$ & 7.0 & 2414.7 & 0.2667 & 0.4282 & Low-middle \\
\hline Syria & $32.4 \%(6,032,616)$ & 3.3 & - & 0.3881 & 0.5790 & Middle \\
\hline Tunisia & $22.6 \%(2,546,994)$ & 1.4 & 3872.5 & 0.4503 & 0.6515 & Middle \\
\hline United Arab Emirates & $16.6 \%(1,516,072)$ & 0.8 & $40,438.8$ & 0.6324 & 0.8747 & High \\
\hline Yemen & $34.2 \%(9,191,689)$ & 5.0 & 1406.3 & 0.1329 & 0.4080 & Low-middle \\
\hline Total & $28.4 \%(184,431,081)$ & 100 & - & - & - & - \\
\hline
\end{tabular}

This table details the 22 countries in the East Mediterranean region. It provides the population of adolescents and contribution of each country to the total adolescent population in the Eastern Mediterranean Region. It also provides the overall country-level GDP in 2015 and SDI in 1990 and 2015. Dashes indicate data are unavailable

deteriorate further, with both regional and global consequences for health, social stability, and economic development.

Adolescence is increasingly understood as a key developmental stage for assuring health across the course of one's life, and as such, provides significant opportunities to improve population health in the EMR (Patton et al. 2016; The World Bank 2006). Firstly, adolescents represent more than a quarter of the population in the EMR, and their health needs are likely to be distinct from children and adults. Conflict and civil unrest (which have been a feature of many countries in the EMR) have a large impact on the health of young people, both acutely (through high rates of mortality and morbidity due to violence) but also in the longer term (including mental health disorder and poor sexual and reproductive health) (Viner et al. 2012). Secondly, many health risks typically emerge during adolescence including those for non-communicable diseases (NCDs) such as substance use, overweight, and physical inactivity. Given that NCDs are now the leading cause of poor health in the EMR, there is a potential to intervene before harms arise (Mokdad et al. 2016b). Thirdly, adolescents are critical to driving socioeconomic development (The World Bank 2006). Poor physical health and mental health are barriers to participation in education and employment, as are policies and systems that do not enable equitable access. Finally, in their role as current and future parents, the health of adolescents has significant implications for the next generation (Patton et al. 2016).

To date, the health problems and health risks of adolescents in the EMR have been inadequately described (Alaovie et al. 2017). This is a significant barrier to developing comprehensive policies that address adolescent health and to measuring the impact of any investments made. This paper aims to report the health profile for adolescents living in the EMR.

\section{Methods}

We framed our study around the conceptual framework defined by the Lancet Commission of Adolescent Health and Wellbeing (hereafter referred to as the Commission) 
(Patton et al. 2016). Health needs included: health outcomes (mortality, non-fatal diseases, and injuries); health risks (behaviors and states that carry risk for poor health in and beyond adolescence); and determinants of health (such as education and employment). Adolescence was defined as 10-24 years, as these years encompass the important biological, neurocognitive, and social role transitions that typically define adolescence (Mokdad et al. 2016b; Patton et al. 2016; Sawyer et al. 2012). Where possible, we report age-disaggregated data for young adolescents (10-14 years), older adolescents (15-19 years), and young adults (20-24 years) (Patton et al. 2016).

Data are drawn from the Global Burden of Disease Study 2015 (GBD 2015) as this provides a complete set of comparable health estimates for 195 countries, including all those in the EMR. Methods are described in detail elsewhere (GBD DALYs Hale Collaborators 2016; GBD Disease Injury Incidence and Prevalence Collaborators 2016; GBD Mortality and Causes of Death Collaborators 2016; GBD SDG Collaborators 2016), but briefly, GBD 2015 includes a comprehensive and systematic analysis of 249 causes of death, 310 causes of disease and injury, and 79 behavioral and environmental health risks. GBD 2015 has four levels of causes that are mutually exclusive. Level one has three causes: type I conditions (communicable, maternal, neonatal, and nutritional disorders); non-communicable diseases; and injuries. Level two has 21 causes, while levels three and four consist of all disaggregated causes. For this analysis we report causes at level four. GBD is based on the best available primary data and employs a series of disease models to harmonize health estimates and fill data gaps. Each step of the estimation process of GBD 2015 has been documented, as well as data sources, in accordance with Guidelines for Accurate and Transparent Health Estimates Reporting (GATHER). For this analysis, we accessed data in 5-year age bands, for males and females, from 1990 to 2015 in 5-year time slices.

Mortality is reported as all-cause and cause-specific rates per 100,000 (GBD Mortality and Causes of Death Collaborators 2016). Non-fatal diseases and injuries are reported as years lived with disability (YLDs), a metric which incorporates prevalence of disease, duration, and its severity (using disease weights) (GBD Disease Injury Incidence and Prevalence Collaborators 2016). As a summary measure of population health, we also report disability-adjusted life-years (DALYs), the sum of years of healthy life lost due to premature mortality (YLLs), and years of life lived with disability (YLDs) (GBD DALYs Hale Collaborators 2016). For these estimates, we report 95\% uncertainty estimates, which are distinct from confidence intervals in that they represent uncertainty derived from sampling, model estimation, and model specification (GBD DALYs Hale Collaborators 2016).
In addition to region-level estimates, we also report country-specific DALY estimates and the prevalence of three key health risks and four determinants (aligned with the conceptual framework from the Commission and data availability) to help prioritize country-specific actions (Patton et al. 2016). Data for health risks were sourced from GBD 2015. Tobacco smoking was defined as current daily smoked tobacco use (GBD Tobacco Collaborators 2017). Overweight was defined using the International Obesity Task Force age and gender specific cut-offs, equivalent to $\mathrm{BMI} \geq 25 \mathrm{~kg} / \mathrm{m}^{2}$ at age 18 (Cole and Lobstein 2012). This definition includes those who are obese. Binge drinking was defined as having consumed 60 grams of alcohol on a single occasion for males and 48 grams of alcohol on a single occasion for females in the last 12 months. With respect to determinants, adolescent fertility rate (live births per 1000 15- to 19-year-old females) and mean years of educational attainment for 15- to 24-year olds were sourced from GBD 2015 (GBD SDG Collaborators 2016). Unmet need for contraception (15- to 24-year-old females currently married or in union and not wanting to become pregnant within the next two years, who report not using any method of contraception) was sourced from a review DHS and MICS surveys available in the EMR (data were collected from 2009 to 2014) (Patton et al. 2016). Youth unemployment data, defined as the percentage of 15- to 24-year olds without work but available for and seeking employment, were obtained from the International Labor Organization modeled estimates for 2013 (Patton et al. 2016).

We reported observed estimates for the region. We additionally report expected DALYs for each country based on the level of socioeconomic development. Expected DALYs were estimated using the Socio-demographic Index (SDI) which is based on income per capita, average educational attainment for ages 15 or older, and the total fertility rate (GBD SDG Collaborators 2016). SDI is reported as a continuous variable from 0 (lowest) to 1 (highest), and as quintiles, as shown in Table 1. GBD 2015 has estimated the relationship between SDI and each cause of DALYs using spline regressions, with these regressions then used to estimate expected DALYs at each level of SDI (GBD DALYs Hale Collaborators 2016).

\section{Results}

\section{Mortality}

All-cause mortality rates for adolescents in the EMR ranged from 63.3 per 100,000 for females aged 10-14 years to 253.2 per 100,000 for males aged 20-24 years in 2015 (e-Figure 1, panel A). Males had a 
higher overall mortality rate than females. The risk of mortality for males aged 15-24 years had increased over recent years compared to what was otherwise an overall trend of reduction in mortality. Table 2 details the leading causes of mortality by sex. The most striking transition in mortality cause over time was the reduction in deaths due to natural disaster and communicable diseases, and the emergence of mortality due to injuries (especially in the context of war) and NCDs. In 1990 and 2005 natural disaster ranked as a leading cause of mortality for adolescents, whereas in 2015 it was no longer a leading cause of death for adolescents. In 2015, war and legal interventions (law enforcement) was the leading cause of death for adolescents of both sexes, representing $27.7 \% \quad(14.2-38.4)$ of deaths amongst male 20 - to 24 -year olds and $7.2 \%$ (3.1-10.9) amongst female 20- to 24 -year olds. For males, injuries (unintentional injuries, self-harm and violence) were the predominant causes of mortality across adolescence, and communicable diseases an important cause for 10to 14-year olds. The leading cause of mortality for females included injuries; however, communicable and maternal conditions were also leading causes, with NCDs emerging as an important cause amongst older female adolescents.

\section{YLDs}

All-cause YLD rates are similar for males and females in the region, and have seen little improvement since 1990 (eFigure 1 panel B). The leading causes of YLDs are detailed in Table 3. While the burden of some communicable, maternal, and nutritional disorders has declined, this has largely been offset by an increase in disability due to injury among males and lack of reduction in YLDs from noncommunicable disease. From 1990 to 2015, iron deficiency anemia was the leading cause of disability for females aged 10-14 and 15-19 years, and for males aged 10-14 years. NCDs, particularly mental health disorders, migraine, asthma, skin conditions, and musculoskeletal disorders, were major contributors to YLDs for both sexes in 2015 . Major depression emerged as the leading cause of morbidity amongst males aged 15-19 (7.0\%, uncertainty 4.6-9.9) and 20-24 years (8.0\%, uncertainty 5.1-11.7) and for females aged 20-24 years $(9.1 \%$, uncertainty $6.2-12.4)$ in 2015 . Among older males, opioid use disorders and war were also important causes of disability.

\section{DALYs}

All-cause DALY rates have declined for females of all ages and 10- to 14-year-old males in the region (e-Figure 1, panel c), largely due to a reduction of communicable, maternal, and nutritional diseases. DALY rates have increased for males aged 15-19 and 20-24 since 1990, largely due to an increased burden of injury. The leading causes of DALYs by age and sex are provided in Table 4. For females, type 1 conditions (nutritional disorders, communicable disease, and maternal disorders) remain important causes of DALYs. However, non-communicable diseases (mental health disorders, migraine, skin conditions, and musculoskeletal disorders) account for more than half of the total disease burden. For males, injuries due to conflict, transport, and other unintentional injuries are the leading causes of DALYs, particularly amongst 15 - to 24-year olds.

There was considerable variation in all-cause and cause-specific DALY rates across countries in the region (e-Figure 2, panels A-C). In all countries in the region, DALY rates are highest among males, and higher among 20- to 24-year olds than other ages in males and females. The highest DALY rates were in countries most affected by recent conflict or insecurity and/or those with the lowest SDI, such as Pakistan (17,483 per 100,000 10-24 years in 2015), Somalia (27,716 DALYs per 100,000 10- to 24-year olds in 2015), Afghanistan (32,068 per 100,000 10- to 24-year olds in 2015) and Syria (33,452 per 100,000 10- to 24-year olds in 2015), with 10- to 24-year olds globally having a DALY rate of 14,557 per 100,000 in 2015 . In these countries, injury, particularly due to war and legal intervention was a major contributor to DALYs, particularly amongst males. For example, in Syria over $70 \%$ of DALYs to $10-24$ year olds were due to injury, with males aged 20-24 experiencing the largest burden. A number of these countries also experience a high burden of nutritional disorders and communicable disease among younger adolescents, in addition to a substantial burden of maternal health problems. Countries with a higher SDI and those less affected by conflict experienced a lower burden of poor health. In these settings DALYs were mostly due to NCDs including mental health disorders, skin conditions, asthma, migraine, and musculoskeletal disorders.

The three countries that had the largest populations of adolescents in the EMR (Pakistan, Iran, and Egypt) had very different disease burdens. Egypt (12,418 DALYs per 100,000 10- to 24-year olds in 2015) and Iran (12,624 DALYs per 100,000 10- to 24-year olds in 2015) has similar low rates of DALYs; however, Iran had a higher burden due to injury (3361 per 100,000 in Iran compared to 1938 per 100,000 in Egypt). Pakistan had a high burden of injury (3367 per $100,000)$ but more so type I conditions (5252 per 100,000).

e-Figure 2, panels D-F shows the expected DALYs in each country based on the SDI. The most striking finding is that with the reduction of DALYs due to war (which is not expected based on development), there remains a very large burden of poor health for adolescents in the EMR. 


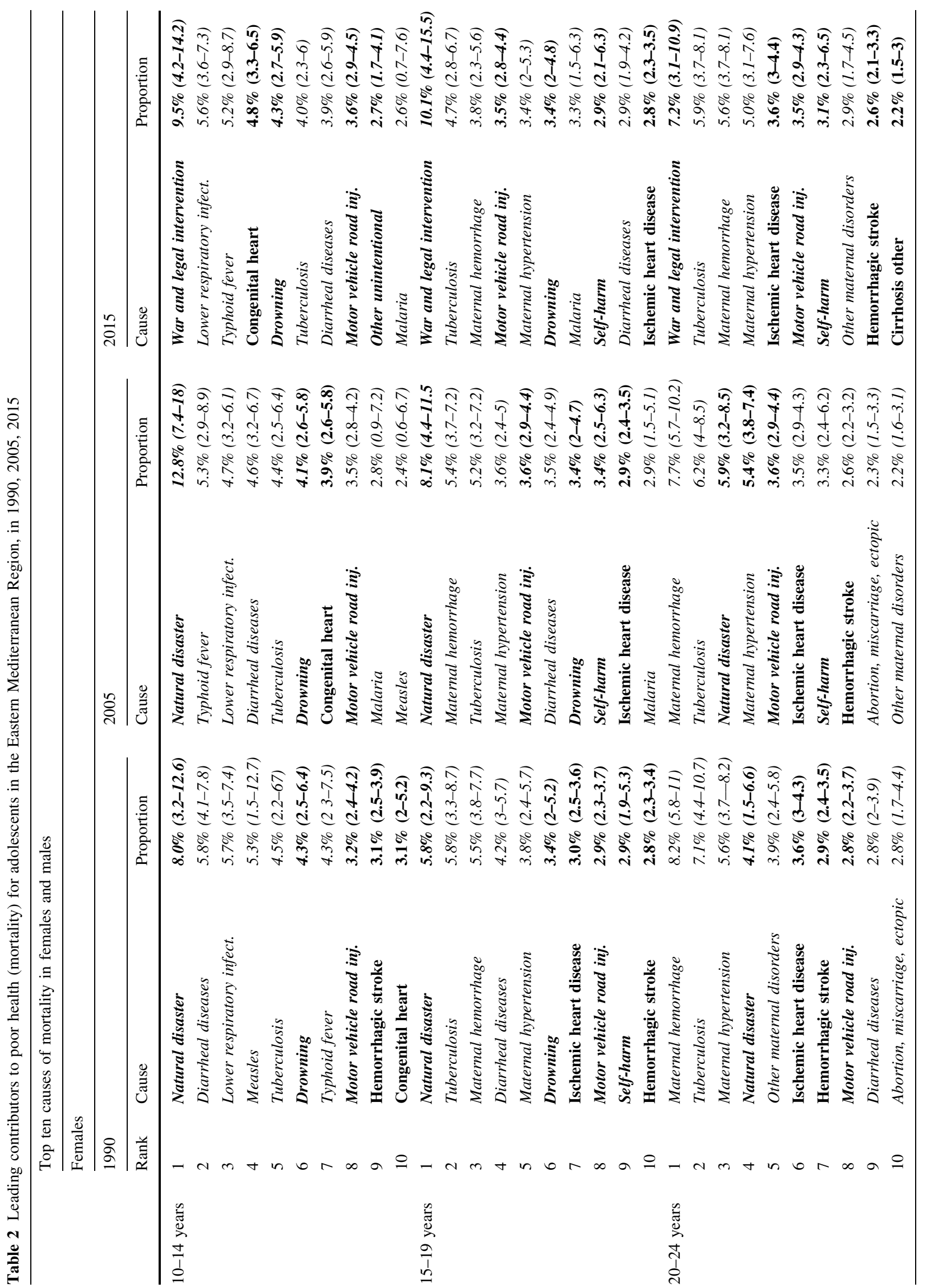




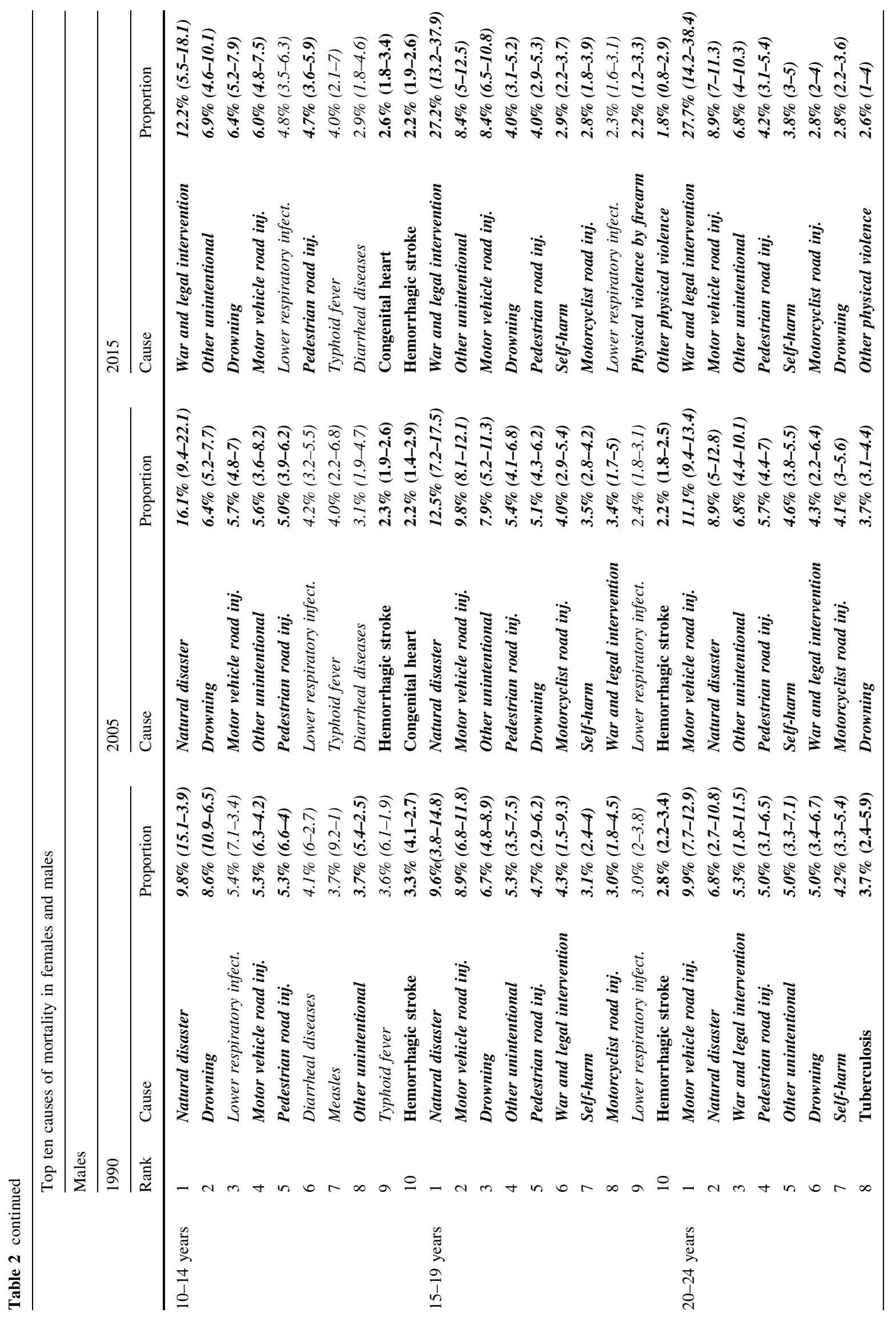




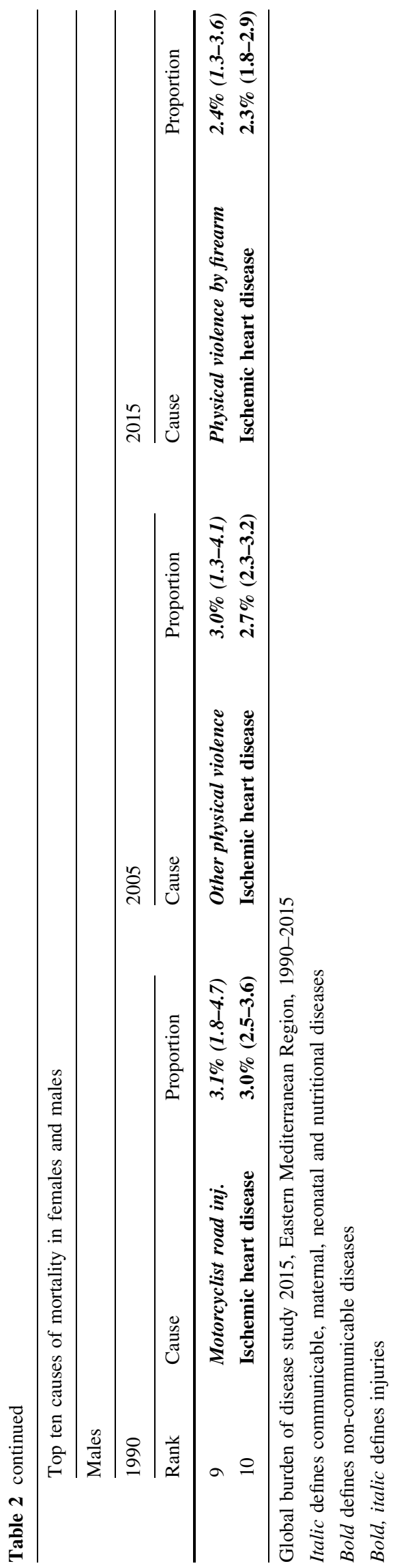

\section{Health risks and determinants}

The prevalence of selected health risks is provided in e-Figure 3 . The prevalence of overweight and obesity was highest among countries with a higher SDI in the region, and was generally similar for males and females. Rates of daily tobacco smoking among males aged 10-24 years ranged from $1.9 \%$ in Sudan to $18 \%$ in Kuwait, but were less than $5 \%$ for females in the region. Similarly, the prevalence of binge drinking was higher among males than females at all ages, but was less than $10 \%$ for both sexes in most EMR countries. Unmet need for contraception was high among the 11 countries for which data are available (e-Figure 4). More than one third of females who are married or in union have unmet need for contraception in Pakistan, Djibouti, Somalia, Sudan, and Yemen. These countries also have among the highest rates of adolescent birth rates in the region, adolescent fertility the greatest in Somalia (114.7 live births per 1000 females aged 15-19 in 2015). There was also great variation in educational attainment in the EMR region. Low-SDI countries affected by protracted insecurity and conflict have the lowest mean number of years of completed education, most notably for females. Rates of unemployment among 15- to 24-year olds also vary considerably, and were generally higher for females than males.

\section{Discussion}

This study is the first systematic analysis of adolescent health in the EMR. The findings suggest dramatic shifts in the health of adolescents living in the EMR over the past 25 years. Communicable diseases and the health consequences of natural disasters have reduced substantially, but these gains have largely been offset by war and the emergence of NCDs including mental health disorders, unintentional injury, and self-harm. Indeed, adolescents living in Syria, Afghanistan, and Somalia experience amongst the largest burdens of disease and injury of all adolescents globally (Patton et al. 2016). Even with the return of peace and security to this region, adolescents will have a persisting poor health profile that will pose a barrier to socioeconomic growth and development of the EMR (The World Bank 2006).

The substantial reductions in mortality and morbidity due to communicable disease, maternal disorders, and natural disasters in the EMR are likely the result of socioeconomic growth and development, educational participation, and interventions through the health system. Recent wars and civil conflict, however, threaten the foundations on which these gains were made, with the risk of resurgence of many of these conditions. This is in 


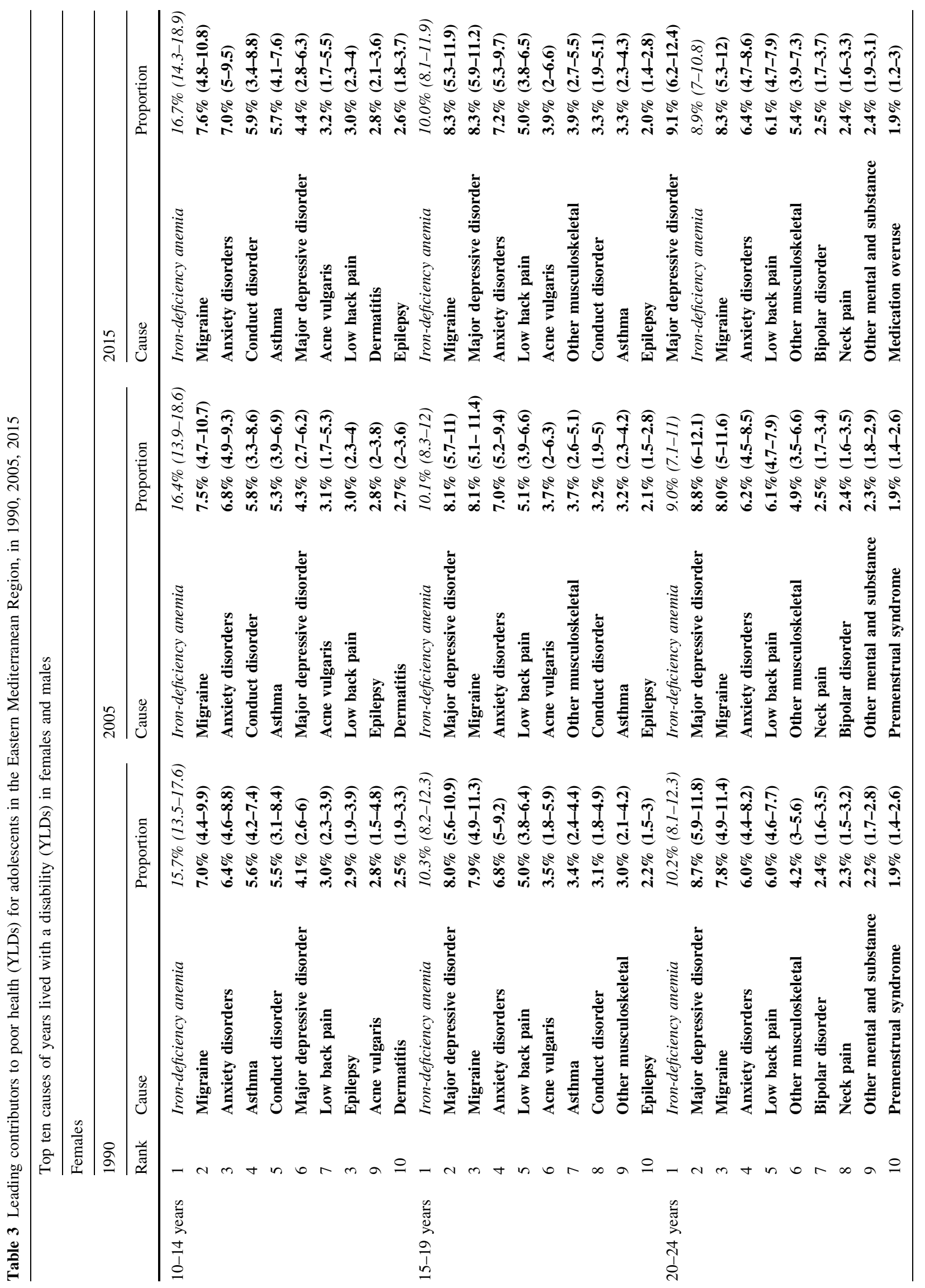




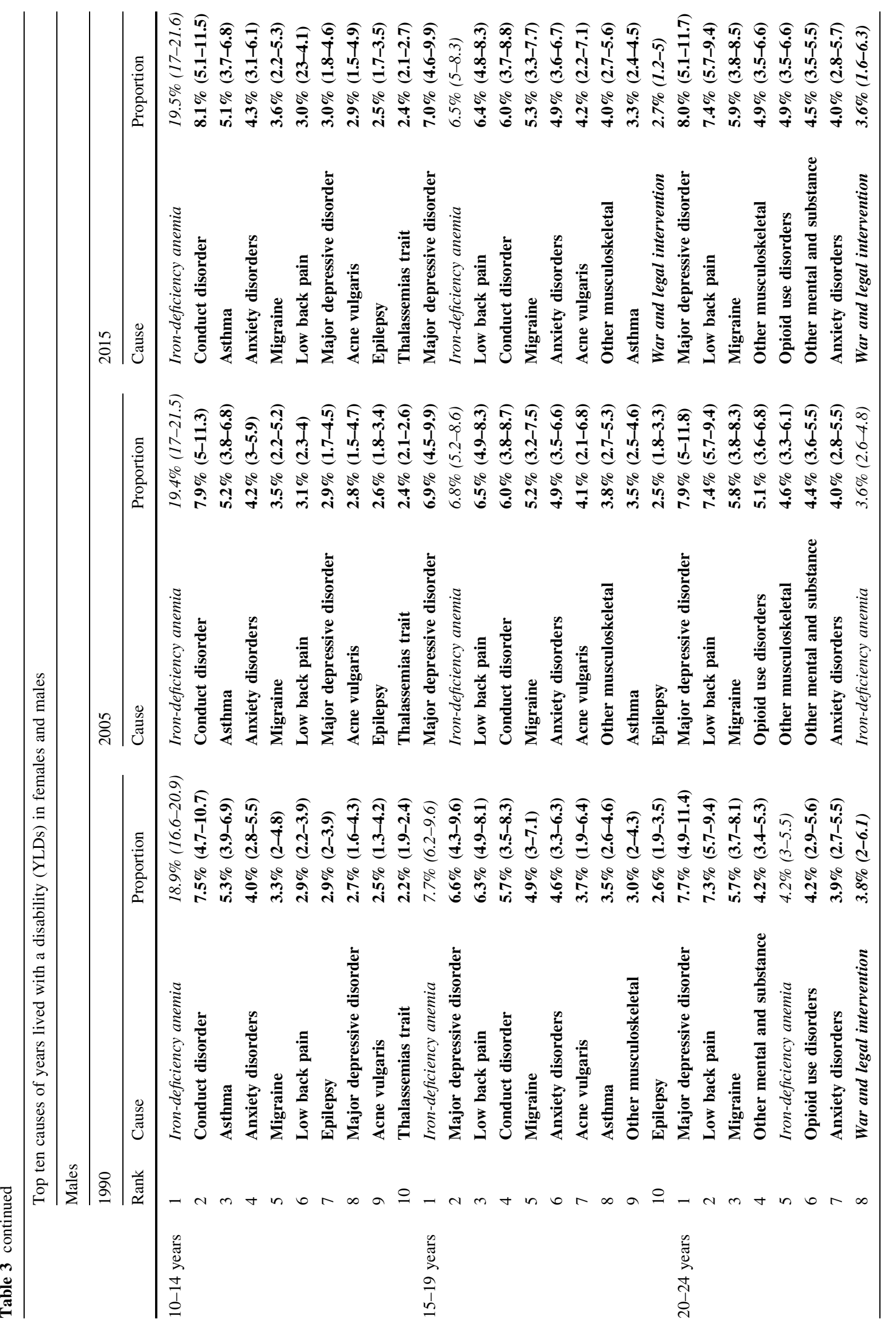




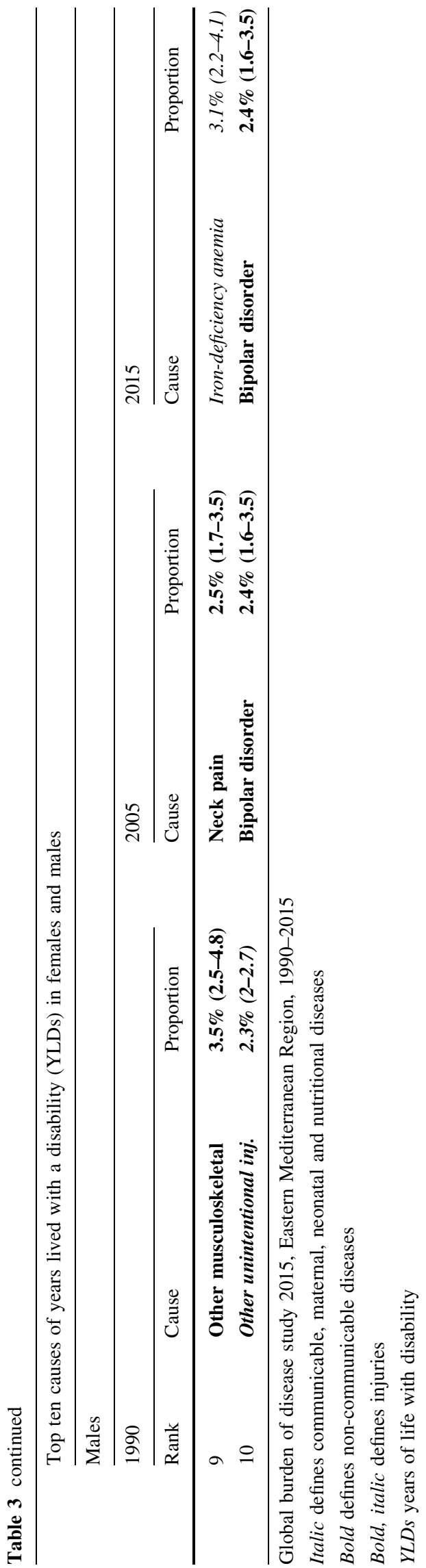

addition to the growing burden of NCDs and injuries. Renewed efforts to consolidate gains made in communicable disease and maternal health will be required. The Lancet Commission described the benefits that can accrue from investments in adolescence. Without these investments, the EMR region risks poor health across adolescence, adulthood, and in the next generation (Patton et al. 2016).

War and legal interventions (deaths due to law enforcement, regardless of their legality) were the leading causes of mortality for adolescents aged 10-24 years of both genders in the EMR. While not all countries in the EMR are affected by conflict, the magnitude of mortality in those countries that are affected signifies this as a priority for the region. It should also be noted, however, that at a population level (all ages combined), war and legal intervention is only the fifth leading cause of mortality in the EMR (GBD 2015 Eastern Mediterranean Region Collaborators and Mokdad 2017). This may reflect competing causes of mortality at other ages, or it may signify that war and legal interventions disproportionately affect adolescents, particularly 20- to 24-year-old males. In addition to the physical injuries and disability that accompany conflict, violence and trauma at this critical developmental stage carry a risk of persisting effects on future mental health and well-being. War and conflict are also likely to result in disruption to quality education, social infrastructure, and community development, which have profound implications for health and well-being across the course of one's life. This may explain why expected DALYs in countries which have experienced large burdens of conflict remain high.

In addition to the health status of adolescents, we explored the burden of risk behavior for future health. Tobacco smoking and high body mass were common risks where interventions during adolescence have the potential to avert later ischemic heart disease, the leading cause of poor health in the region. Of note, estimates of tobacco smoking reported here do not include Sisha smoking which is prevalent in some countries of the EMR and harmful (Maziak et al. 2004). Low rates of education completion in many countries including Afghanistan, Somalia, and Yemen are a major obstacle to growth and future prosperity of this region. High rates of adolescent pregnancy represent an important target for action to improve health and life opportunities for girls and young women. Unmet need for contraception is very high, and is likely to also signify high rates of unmet need for other essential health interventions, particularly for culturally sensitive needs such as sexually transmitted infections and mental health. Given adolescents face barriers in accessing health facilities, there is a need to explore other approaches such as community-based delivery or school health services (Tylee et al. 2007). There is 


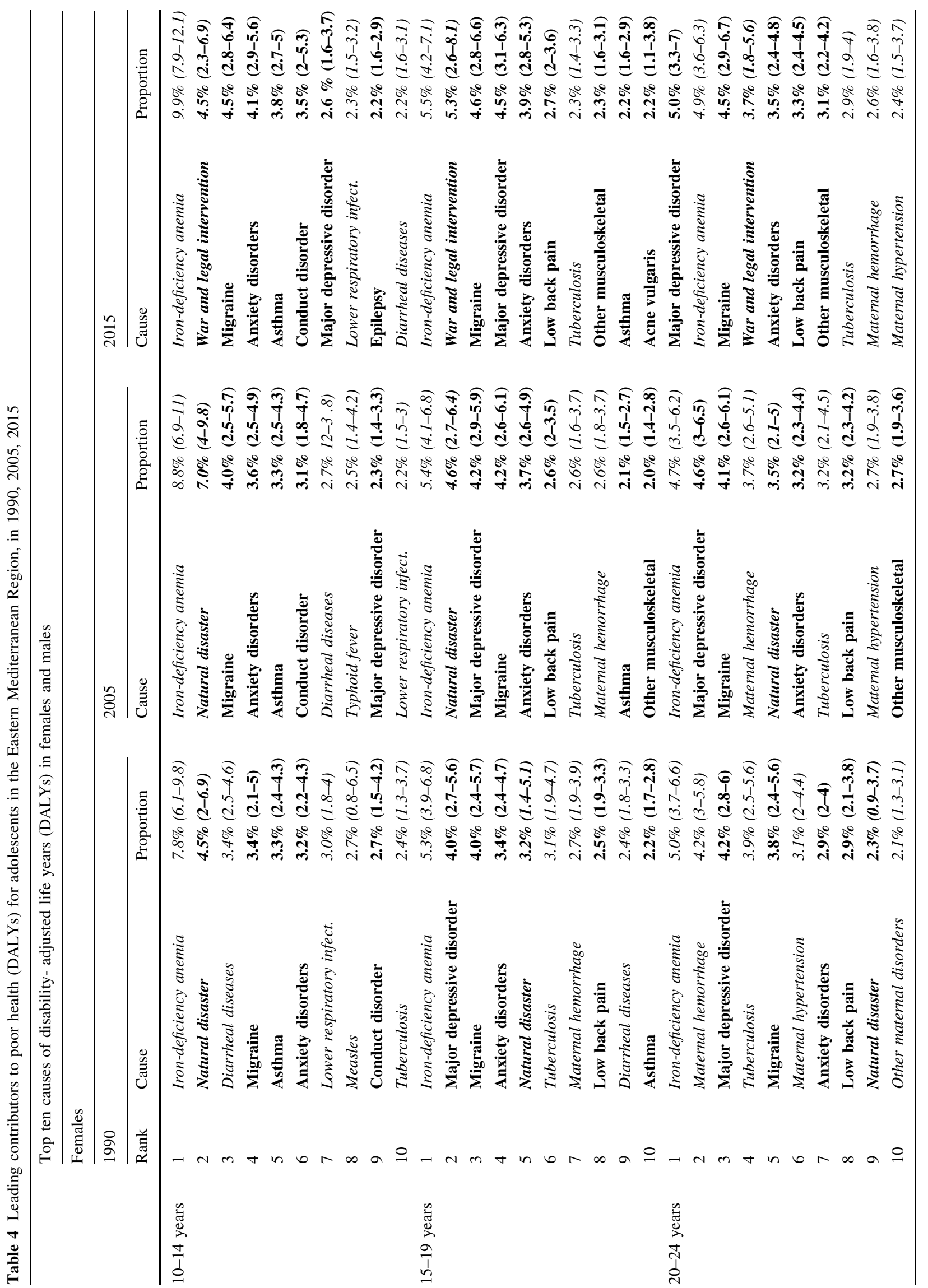




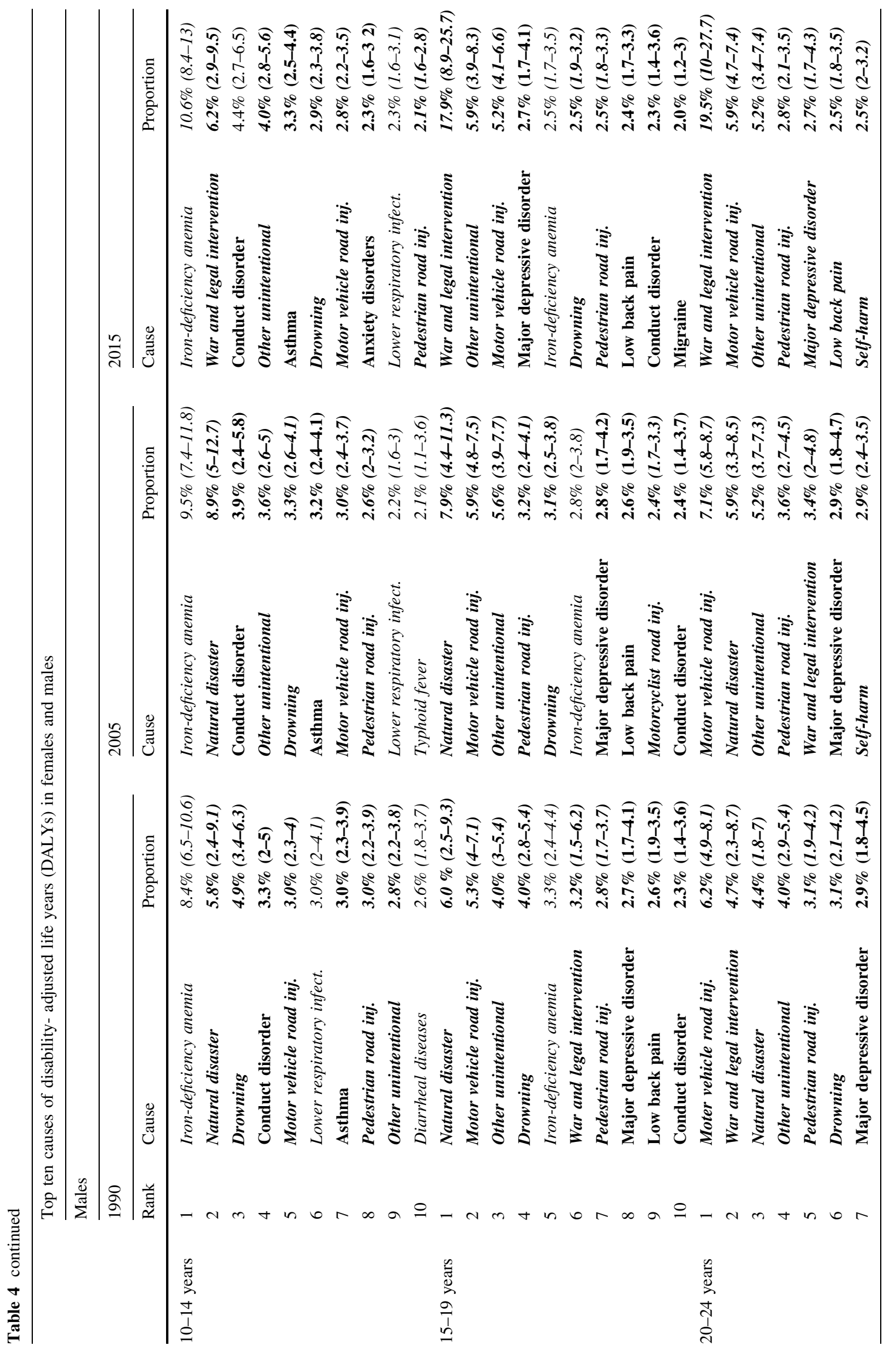




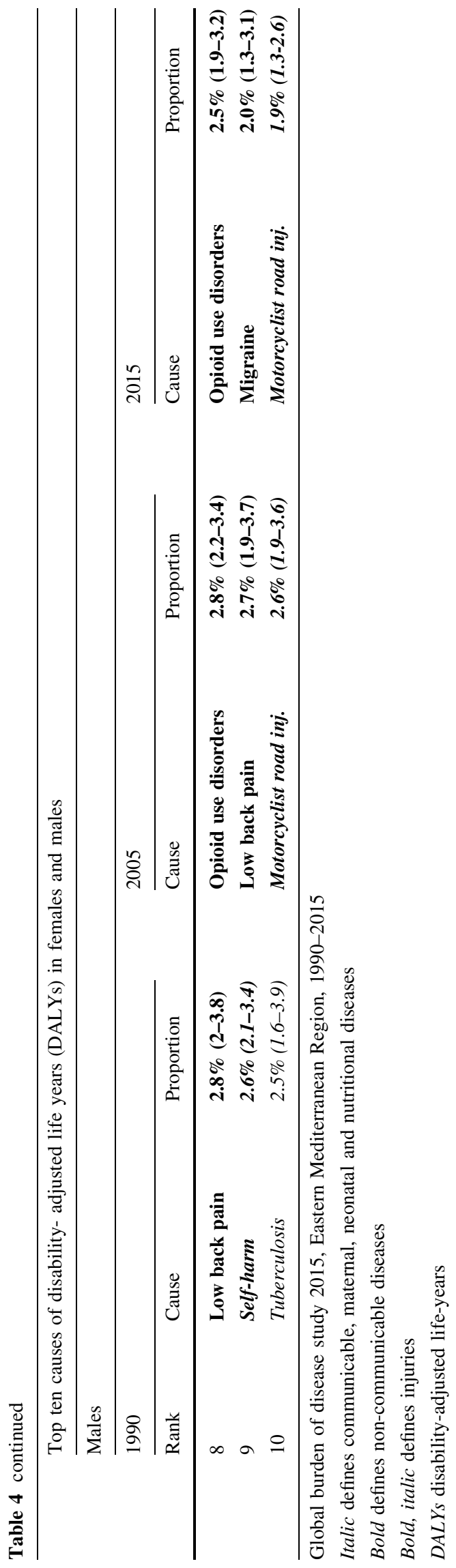

also the need for community-based interventions to address some of the sociocultural barriers that contribute to high unmet need for interventions relating to sexual and reproductive health (Patton et al. 2016).

As over half of the region's adolescents live in Pakistan, Iran, and Egypt (Table 1), a regional response must prioritize these three countries. This is challenging, however, given the different stages of development and the very different needs of adolescents across these countries. For example, the burden of disease experienced by adolescents in Egypt is predominantly caused by NCDs, which require interventions to address the burden of chronic illness and health risks such as tobacco smoking and obesity (Patton et al. 2016). In addition to NCDs, adolescents living in Iran are burdened by injury (particularly relating to transport injury) which requires a suite of inter-sectoral actions (WHO 2017a). Adolescents living in Pakistan experience a "multi-burden" profile of disease, with a large burden of communicable, nutritional, and reproductive poor health in addition to NCDs and injury. However, in countries such as Afghanistan, Somalia, and Sudan, adolescents represent almost a third of the country-level population and experience a particularly large and complex burden of disease. It is, therefore, important not to neglect their health needs (or indeed other countries in the EMR).

What this analysis highlights is that unintentional injury, mental health, sexual health, substance use, and self-harm are increasingly important health issues for adolescents in the EMR. While these are health issues common to adolescents globally (Patton et al. 2016), they have typically sat at the margins (if at all) of policy, program, and data collection in EMR, given religious and cultural sensitivities. These findings highlight the need to better align health actions, including data monitoring of sensitive health outcomes, including risks, in this region to these needs.

Our analysis has important limitations. Firstly, there is considerable variation in the availability and quality of primary data for adolescent health. This includes paucity of data for some age groups (particularly 10- to 14-year olds), and for many health outcomes and risks of importance during these important developmental years (Mokdad et al. 2016b; Patton et al. 2012; The Global Burden of Disease Child and Adoelscent Health Collaboration 2017). Availability of timely, quality data is likely to be particularly poor in settings of conflict and insecurity, which affects many countries in this region. The poor quality of primary data necessitated the use of modeled estimates, and some of these modeled estimates may have over- or under-estimated the true burden. For example, ischemic heart disease was found to be a leading cause of mortality amongst males aged 20-24 years, a cause of death more commonly associated with adulthood. Premature death due to 
cardiovascular disease is possible during adolescence, particularly in the context of adolescent obesity which is prevalent in EMR (Franks et al. 2010). This finding may also be an artifact of disease modeling, as ischemic heart disease is the leading cause of mortality in the EMR, and these deaths are modeled to have their onset after 0.1 years of age (GBD 2015 Eastern Mediterranean Region Cardiovascular Disease Collaborators and Mokda 2017; GBD 2015 Eastern Mediterranean Region Collaborators and Mokdad 2017; GBD Mortality and Causes of Death Collaborators 2016). The findings of this study should therefore be interpreted as not only indicating priority areas to address adolescent health in the EMR, but also where data collection efforts should focus. A further limitation is that the broader impacts of armed conflict on adolescent health and well-being, beyond mortality, are not captured by the 2015 GBD study. These include participation in education and employment, as well as the impacts of trauma on adolescent development and wellbeing. Additionally, some important health issues such as female genital cutting/mutilation (common in countries such as Somalia) are not included in GBD 2015 (UNICEF 2016a).

There are several regional efforts that may facilitate addressing the needs of adolescents in the EMR. For example, a coalition of youth advocates for health in the EMR has been established (Alaovie et al. 2017). There is a joint UN strategy for youth in the region (IATTTYP 2015). UNICEF has also published guidance around good practice with adolescent and youth programming (UNICEF 2016b). This study compliments these efforts, and helps to inform some priority areas for health. For conflict-affected countries, the focus must clearly be on the return of peace and stability and the rebuilding of health, education, and social systems. In doing so, it is important to design services that meet the needs of adolescents. For countries not affected by conflict, health actions include the need to re-orientate health systems to focus on prevention and the growing burden of NCDs. This needs to extend to inter-sectoral actions to address the broader determinants of NCDs and injuries. Without urgent action, there is a risk that profiles of adolescent health will continue to deteriorate with consequences for future population health and wellbeing, productivity, and ultimately the stability of civil society.

GBD 2015 Eastern Mediterranean Region Adolescent Health Collaborators: Ali H. Mokdad, PhD (corresponding author), Institute for Health Metrics and Evaluation, University of Washington, Seattle, Washington, United States. Peter Azzopardi, PhD, Burnet Institute, Melbourne, VIC, Australia; Murdoch Children's Research Institute, Melbourne, Victoria, Australia; Wardliparingga Aboriginal Research Unit, South Australian Health and Medical Research Institute (SAHMRI), Adelaide, South Australia, Australia. Karly Cini, MClinEpi, Centre for Adolescent Health, Murdoch Childrens Research Institute, Melbourne, Victoria, Australia. Elissa Kennedy, MBBS, MPH, Burnet Institute, Melbourne, VIC, Australia. Susan
Sawyer, MD, Murdoch Childrens Research Institute, The University of Melbourne, Parkville, Victoria, Australia. Charbel El Bcheraoui, $\mathrm{PhD}$, Institute for Health Metrics and Evaluation, University of Washington, Seattle, Washington, United States. Raghid Charara, MD, American University of Beirut, Beirut, Lebanon. Ibrahim Khalil, MD, Institute for Health Metrics and Evaluation, University of Washington, Seattle, Washington, United States. Maziar MoradiLakeh, MD, Department of Community Medicine, Preventive Medicine and Public Health Research Center, Gastrointestinal and Liver Disease Research Center (GILDRC), Iran University of Medical Sciences, Tehran, Iran. Michael Collison, BS, Institute for Health Metrics and Evaluation, University of Washington, Seattle, Washington, United States. Rima A. Afifi, PhD, American University of Beirut, Beirut, Lebanon. Jamela Al-Raiby, MD, World Health Organization. Kristopher J. Krohn, BA, Institute for Health Metrics and Evaluation, University of Washington, Seattle, Washington, United States. Farah Daoud, BA/BS, Institute for Health Metrics and Evaluation, University of Washington. Adrienne Chew, ND, Institute for Health Metrics and Evaluation, University of Washington, Seattle, Washington, United States. Ashkan Afshin, MD, Institute for Health Metrics and Evaluation, University of Washington, Seattle, Washington, United States. Kyle J. Foreman, PhD, Institute for Health Metrics and Evaluation, University of Washington, Seattle, Washington, United States; Imperial College London, London, United Kingdom. Nicholas J. Kassebaum, MD, Institute for Health Metrics and Evaluation, University of Washington, Seattle, Washington, United States; Department of Anesthesiology \& Pain Medicine, Seattle Children's Hospital, Seattle, Washington, United States. Michael Kutz, BS, Institute for Health Metrics and Evaluation, University of Washington, Seattle, Washington, United States. Hmwe $\mathrm{H}$. Kyu, PhD, Institute for Health Metrics and Evaluation, University of Washington, Seattle, Washington, United States. Patrick Liu, BA, Institute for Health Metrics and Evaluation, University of Washington, Seattle, Washington, United States. Helen E. Olsen, MA, Institute for Health Metrics and Evaluation, University of Washington, Seattle, Washington, United States. Alison Smith, BA, Institute for Health Metrics and Evaluation, University of Washington, Seattle, Washington, United States. Jeffrey D. Stanaway, PhD, Institute for Health Metrics and Evaluation, University of Washington, Seattle, Washington, United States. Haidong Wang, $\mathrm{PhD}$, Institute for Health Metrics and Evaluation, University of Washington, Seattle, Washington, United States. Johan Ärnlöv, PhD, Department of Neurobiology, Care Sciences and Society, Division of Family Medicine and Primary Care, Karolinska Institutet, Stockholm, Sweden; School of Health and Social Studies, Dalarna University, Falun, Sweden. Aliasghar Ahmad Kiadaliri, PhD, Department of Clinical Sciences Lund, Orthopedics, Clinical Epidemiology Unit, Lund University, Lund, Sweden. Khurshid Alam, PhD, Murdoch Childrens Research Institute, The University of Melbourne, Parkville, Victoria, Australia; The University of Melbourne, Melbourne, VIC, Australia; The University of Sydney, Sydney, NSW, Australia. Deena Alasfoor, MSc, Ministry of Health, Al Khuwair, Muscat, Oman. Raghib Ali MSc, University of Oxford, Oxford, United Kingdom. Reza AlizadehNavaei, PhD, Gastrointestinal Cancer Research Center, Mazandaran University of Medical Sciences, Sari, Mazandaran, Iran. Rajaa AlRaddadi, PhD, Joint Program of Family and Community Medicine, Jeddah, Makkah, Saudi Arabia. Khalid A. Altirkawi, MD, King Saud University, Riyadh, Saudi Arabia. Nelson Alvis-Guzman, PhD, Universidad de Cartagena, Cartagena de Indias, Colombia. Nahla Anber, PhD, Mansoura University, Mansoura, Egypt. Carl Abelardo T. Antonio, MD, Department of Health Policy and Administration, College of Public Health, University of the Philippines Manila, Manila, Philippines. Palwasha Anwari, MD, Self-employed, Kabul, Afghanistan. Al Artaman, PhD, University of Manitoba, Winnipeg, Manitoba, Canada. Hamid Asayesh, PhD, Department of Medical Emergency, School of Paramedic, Qom University of Medical 
Sciences, Qom, Iran. Suzanne L. Barker-Collo, PhD, School of Psychology, University of Auckland, Auckland, New Zealand. Neeraj Bedi, MD, College of Public Health and Tropical Medicine, Jazan, Saudi Arabia. Ettore Beghi, MD, IRCCS - Istituto di Ricerche Farmacologiche Mario Negri, Milan, Italy. Derrick A. Bennett, PhD, Nuffield Department of Population Health, University of Oxford, Oxford, United Kingdom. Isabela M. Bensenor, $\mathrm{PhD}$, University of São Paulo, São Paulo, Brazil. Zulfiqar A. Bhutta, PhD, Centre of Excellence in Women and Child Health, Aga Khan University, Karachi, Pakistan; Centre for Global Child Health, The Hospital for Sick Children, Toronto, ON, Canada. Zahid A. Butt, PhD, Al Shifa Trust Eye Hospital, Rawalpindi, Pakistan. Carlos A. Castañeda-Orjuela, MSc, Colombian National Health Observatory, Instituto Nacional de Salud, Bogota, DC, Colombia; Epidemiology and Public Health Evaluation Group, Public Health Department, Universidad Nacional de Colombia, Bogota, Colombia. Ferrán Catalá-López, PhD, Department of Medicine, University of Valencia/INCLIVA Health Research Institute and CIBERSAM, Valencia, Spain; Clinical Epidemiology Program, Ottawa Hospital Research Institute, Ottawa, Canada. Fiona J. Charlson, PhD, School of Public Health, University of Queensland, Brisbane, Queensland, Australia; Institute for Health Metrics and Evaluation, University of Washington, Seattle, Washington, United States; Queensland Centre for Mental Health Research, Brisbane, Queensland, Australia. Hadi Danawi, PhD, Walden University, Minneapolis, Minnesota, United States. Diego De Leo, DSc, Griffith University, Brisbane, Queensland, Australia. Louisa Degenhardt, PhD, National Drug and Alcohol Research Centre, University of New South Wales, Sydney, Australia. Donna Denno, MD, Department of Pediatrics, University of Washington, Seattle, Washington, United States; Department of Global Health, University of Washington, Seattle, Washington. Kebede Deribe, MPH, Brighton and Sussex Medical School, Brighton, United Kingdom; School of Public Health, Addis Ababa University, Addis Ababa, Ethiopia. Don C. Des Jarlais, PhD, Mount Sinai Beth Israel, New York, New York, United States; Icahn School of Medicine at Mount Sinai, New York City, New York, United States. Subhojit Dey, PhD, Indian Institute of Public Health-Delhi, Public Health Foundation of India, Gurgaon, India. Samath D. Dharmaratne, MD, Department of Community Medicine, Faculty of Medicine, University of Peradeniya, Peradeniya, Sri Lanka. Shirin Djalalinia, PhD, Undersecretary for Research \& Technology, Ministry of Health \& Medical Education, Tehran, Iran. Holly E. Erskine, PhD, Queensland Centre for Mental Health Research, Brisbane, QLD, Australia; School of Public Health, University of Queensland, Brisbane, QLD, Australia; Institute for Health Metrics and Evaluation, University of Washington, Seattle, Washington, United States. Seyed-Mohammad Fereshtehnejad, PhD, Department of Neurobiology, Care Sciences and Society (NVS), Karolinska Institutet, Stockholm, Sweden. Alize J. Ferrari, PhD, School of Public Health, University of Queensland, Brisbane, Queensland, Australia; Queensland Centre for Mental Health Research, Brisbane, Queensland, Australia; Institute for Health Metrics and Evaluation, University of Washington, Seattle, Washington, United States. Florian Fischer, PhD, School of Public Health, Bielefeld University, Bielefeld, Germany. Tsegaye Tewelde Gebrehiwot, MPH, Jimma University, Jimma, Ethiopia. Johanna M. Geleijnse, $\mathrm{PhD}$, Division of Human Nutrition, Wageningen University, Wageningen, Netherlands. Philimon N. Gona, PhD, University of Massachusetts Boston, Boston, Massachusetts, United States. Harish Chander Gugnani, PhD, Departments of Microbiology and Epidemiology \& Biostatistics, Saint James School of Medicine, The Quarter, Anguilla. Rajeev Gupta, PhD, Eternal Heart Care Centre and Research Institute, Jaipur, Rajasthan, India. Randah Ribhi Hamadeh, DPhil, Arabian Gulf University, Manama, Bahrain. Samer Hamidi, DrPH, Hamdan Bin Mohammed Smart University, Dubai, United Arab Emirates. Josep Maria Haro, MD, Parc Sanitari Sant Joan de Déu - CIBERSAM, Sant Boi de Llobregat, Spain. Roderick J. Hay,
DM, International Foundation for Dermatology, London, United Kingdom; King's College London, London, United Kingdom. Stephen J. C. Hearps, PGDipBiostat, Child Neuropsychology, Murdoch Childrens Research Hospital, Parkville, VIC, Australia. Delia Hendrie, MA, Centre for Population Health Research, Curtin University, Bentley, WA, Australia. Peter J. Hotez, PhD, College of Medicine, Baylor University, Houston, Texas, United States. Guoqing Hu, PhD, Department of Epidemiology and Health Statistics, School of Public Health, Central South University, Changsha, Hunan, China. Jost B. Jonas, MD, Department of Ophthalmology, Medical Faculty Mannheim, Ruprecht-Karls-University Heidelberg, Mannheim, Germany. André Karch, MD, Epidemiological and Statistical Methods Research Group, Helmholtz Centre for Infection Research, Braunschweig, Germany; Hannover-Braunschweig Site, German Center for Infection Research, Braunschweig, Germany. Seyed M. Karimi, PhD, University of Washington Tacoma, Tacoma, WA, United States. Amir Kasaeian, PhD, Hematology-Oncology and Stem Cell Transplantation Research Center, Tehran University of Medical Sciences, Tehran, Iran; Endocrinology and Metabolism Population Sciences Institute, Tehran University of Medical Sciences, Tehran, Iran. Seifu Kebede, MS, Mekelle University, Mekele, Ethiopia. Andre Pascal Kengne, $\mathrm{PhD}$, South African Medical Research Council, Cape Town, South Africa; University of Cape Town, Cape Town, South Africa. Ejaz Ahmad Khan, MD, Health Services Academy, Islamabad, Pakistan. Ardeshir Khosravi, PhD, Iranian Ministry of Health and Medical Education, Tehran, Iran; Non-communicable Diseases Research Center, Tehran University of Medical Sciences, Tehran, Iran. Jagdish Khubchandani, PhD, Department of Nutrition and Health Science, Ball State University, Muncie, Indiana, United States. Yoshihiro Kokubo, PhD, Department of Preventive Cardiology, National Cerebral and Cardiovascular Center, Suita, Japan. Jacek A. Kopec, $\mathrm{PhD}$, University of British Columbia, Vancouver, BC, Canada. Soewarta Kosen, MD, Center for Community Empowerment, Health Policy and Humanities, National Institute of Health Research \& Development, Jakarta, Daerah Khusus Ibukota (DKI) Jakarta, Indonesia. Heidi J. Larson, PhD, Department of Infectious Disease Epidemiology, London School of Hygiene \& Tropical Medicine, London, United Kingdom; Institute for Health Metrics and Evaluation, University of Washington, Seattle, Washington, United States. Anders Larsson, PhD, Department of Medical Sciences, Uppsala University, Uppsala, Sweden. Janet L. Leasher, OD, College of Optometry, Nova Southeastern University, Fort Lauderdale, Florida, United States. Janni Leung, PhD, School of Public Health, University of Queensland, Brisbane, QLD, Australia; University of Washington, Seattle, Washington, United States. Yongmei Li, PhD, San Francisco VA Medical Center, San Francisco, California, United States. Paulo A. Lotufo, DrPH, University of São Paulo, São Paulo, Brazil. Raimundas Lunevicius, $\mathrm{PhD}$, Aintree University Hospital National Health Service Foundation Trust, Liverpool, United Kingdom; School of Medicine, University of Liverpool, Liverpool, United Kingdom. Hassan Magdy Abd El Razek, MBBCH, Mansoura Faculty of Medicine, Mansoura, Egypt. Reza Majdzadeh, PhD, Knowledge Utilization Research Center and Community Based Participatory Research Center, Tehran University of Medical Sciences, Tehran, Iran. Azeem Majeed, MD, Department of Primary Care \& Public Health, Imperial College London, London, England, United Kingdom. Peter Memiah, $\mathrm{PhD}$, University of West Florida, Pensacola, FL, United States. Ziad A. Memish, MD, Saudi Ministry of Health, Riyadh, Saudi Arabia; College of Medicine, Alfaisal University, Riyadh, Saudi Arabia. Walter Mendoza, MD, United Nations Population Fund, Lima, Peru. Francis Apolinary Mhimbira, MS, Ifakara Health Institute, Bagamoyo, Tanzania. Ted R. Miller, PhD, Pacific Institute for Research \& Evaluation, Calverton, MD, United States; Centre for Population Health, Curtin University, Perth, WA, Australia. Philip B. Mitchell, MD, University of New South Wales, Kensington, New South Wales, Australia. Lorenzo Monasta, DSc, Institute for Maternal and Child 
Health, IRCCS "Burlo Garofolo", Trieste, Italy. Carla Makhlouf Obermeyer, DSc, Center for Research on Population and Health, Faculty of Health Sciences, American University of Beirut, Beirut, Lebanon. In-Hwan Oh, PhD, Department of Preventive Medicine, School of Medicine, Kyung Hee University, Seoul, South Korea. Bolajoko Olubukunola Olusanya, PhD, Center for Healthy Start Initiative, Lagos, Lagos, Nigeria. Alberto Ortiz PhD, IIS-Fundacion Jimenez Diaz-UAM, Madrid, Spain. Eun-Kee Park, PhD, Department of Medical Humanities and Social Medicine, College of Medicine, Kosin University, Busan, South Korea. Matti Parry, MD, Reproductive Health and Reseearch/Adolescents and at-risk populations (RHR/ AGH), World Health Organization, Geneva, Switzerland. David M. Pereira, PhD, REQUIMTE/LAQV, Laboratório de Farmacognosia, Departamento de Química, Faculdade de Farmácia, Universidade do Porto, Porto, Portugal. Michael Robert Phillips, MD, Shanghai Jiao Tong University School of Medicine, Shanghai, China; Emory University, Atlanta, Georgia, United States. Farshad Pourmalek, PhD, University of British Columbia, Vancouver, British Columbia, Canada. Mostafa Qorbani, PhD, Non-communicable Diseases Research Center, Alborz University of Medical Sciences, Karaj, Iran. Amir Radfar, MD, A T Still University, Kirksville, MO, United States. Anwar Rafay, MS, Contech International Health Consultants, Lahore, Pakistan; Contech School of Public Health, Lahore, Pakistan. Vafa Rahimi-Movaghar, MD, Sina Trauma and Surgery Research Center, Tehran University of Medical Sciences, Tehran, Iran. Rajesh Kumar Rai, MPH, Society for Health and Demographic Surveillance, Suri, India. Saleem M. Rana, PhD, Contech School of Public Health, Lahore, Punjab, Pakistan; Contech International Health Consultants, Lahore, Pakistan. David Laith Rawaf, MD, WHO Collaborating Centre, Imperial College London, London, United Kingdom; North Hampshire Hospitals, Basingstroke, United Kingdom; University College London Hospitals, London, United Kingdom. Salman Rawaf, MD, Imperial College London, London, United Kingdom. Nicola Reavley, PhD, Melbourne School of Population and Global Health, The University of Melbourne, Melbourne, VIC, Australia. Andre M. N. Renzaho, PhD, Western Sydney University, Penrith, NSW, Australia. Satar Rezaei, PhD, School of Public Health, Kermanshah University of Medical Sciences, Kermanshah, Iran. Mohammad Sadegh-Rezai, MD, Infectious Diseases Research Centre with Focus on Nosocomial Infection, Mazandaran University of Medical Sciences, Sari, Iran. Diego Rios-Zertuche, MPA, Salud Mesoamerica 2015 Initiative, Panama, Panama. Gholamreza Roshandel, PhD, Golestan Research Center of Gastroenterology and Hepatology, Golestan University of Medical Sciences, Gorgan, Iran; Digestive Diseases Research Institute, Tehran University of Medical Sciences, Tehran, Iran. Dietrich Rothenbacher, MD, Institute of Epidemiology and Medical Biometry, Ulm University, Ulm, Germany. Mahdi Safdarian, MD, Sina Trauma \& Surgery Research Center, Tehran University of Medical Sciences, Tehran, Iran. Sare Safi, MS, Ophthalmic Epidemiology Research Center, Shahid Beheshti University of Medical Sciences, Tehran, Iran. Saeid Safiri, PhD, Managerial Epidemiology Research Center, Department of Public Health, School of Nursing and Midwifery, Maragheh University of Medical Sciences, Maragheh, Iran. Mohammad Ali Sahraian, MD, MS Research Center, Neuroscience Institute, Tehran University of Medical Sciences, Tehran, Iran. Payman Salamati, MD, Sina Trauma and Surgery Research Center, Tehran University of Medical Sciences, Tehran, Iran. Abdallah M. Samy, PhD, Ain Shams University, Cairo, Egypt. Juan Ramon Sanabria, MD, J Edwards School of Medicine, Marshall Univeristy, Huntington, WV, United States; Case Western Reserve University, Cleveland, OH, United States. Damian Santomauro, PhD, School of Public Health, University of Queensland, Brisbane, Queensland, Australia; Queensland Centre for Mental Health Research, Brisbane, Queensland, Australia; Institute for Health Metrics and Evaluation, University of Washington, Seattle, Washington, United States. Benn Sartorius, PhD, Public Health Medicine, School of Nursing and Public Health, University of KwaZulu-Natal, Durban, South Africa; UKZN Gastrointestinal Cancer Research Centre, South African Medical Research Council (SAMRC), Durban, South Africa. David C. Schwebel, PhD, University of Alabama at Birmingham, Birmingham, Alabama, United States. Soraya Seedat, PhD, Stellenbosch University, Cape Town, South Africa. Sadaf G. Sepanlou, PhD, Digestive Diseases Research Institute, Tehran University of Medical Sciences, Tehran, Iran. Tesfaye Setegn, MPH, Bahir Dar University, Bahir Dar, Ethiopia. Amira Shaheen, PhD, Department of Public Health, An-Najah University, Nablus, Palestine. Masood Ali Shaikh, MD, Independent Consultant, Karachi, Pakistan. Rahman Shiri, PhD, Finnish Institute of Occupational Health, Work Organizations, Work Disability Program, Department of Public Health, Faculty of Medicine, University of Helsinki, Helsinki, Finland. Inga Dora Sigfusdottir, PhD, Reykjavik University, Reykjavik, Iceland. Jasvinder A. Singh, MD, University of Alabama at Birmingham and Birmingham Veterans Affairs Medical Center, Birmingham, Alabama, United States. Badr H. A. Sobaih, MD, King Saud University, Riyadh, Saudi Arabia. Chandrashekhar T. Sreeramareddy, MD, Department of Community Medicine, International Medical University, Kuala Lumpur, Malaysia. Rizwan Suliankatchi Abdulkader, MD, Ministry of Health, Kingdom of Saudi Arabia, Riyadh, Saudi Arabia. Arash Tehrani-Banihashemi, PhD, Preventive Medicine and Public Health Research Center, Iran University of Medical Sciences, Tehran, Iran. Mohamad-Hani Temsah, MD, King Saud University, Riyadh, Saudi Arabia. Abdullah Sulieman Terkawi, MD, Department of Anesthesiology, University of Virginia, Charlottesville, VA, United States; Department of Anesthesiology, King Fahad Medical City, Riyadh, Saudi Arabia; Outcomes Research Consortium, Cleveland Clinic, Cleveland, OH, United States. Alan J. Thomson, PhD, Adaptive Knowledge Management, Victoria, BC, Canada. Marcello Tonelli, MD, University of Calgary, Calgary, Alberta, Canada. Roman ToporMadry, PhD, Institute of Public Health, Faculty of Health Sciences, Jagiellonian University Medical College, Kraków, Poland; Faculty of Health Sciences, Wroclaw Medical University, Wroclaw, Poland. Bach Xuan Tran, PhD, Johns Hopkins University, Baltimore, Maryland, United States; Hanoi Medical University, Hanoi, Vietnam. Kingsley Nnanna Ukwaja, MD, Department of Internal Medicine, Federal Teaching Hospital, Abakaliki, Nigeria. Olalekan A. Uthman, $\mathrm{PhD}$, Warwick Medical School, University of Warwick, Coventry, United Kingdom. Tommi Vasankari, PhD, UKK Institute for Health Promotion Research, Tampere, Finland. Narayanaswamy Venketasubramanian, MBBS, Raffles Neuroscience Centre, Raffles Hospital, Singapore, Singapore. Vasiliy Victorovich Vlassov, MD, National Research University Higher School of Economics, Moscow, Russia. Stein Emil Vollset, DrPH, Center for Disease Burden, Norwegian Institute of Public Health, Bergen, Norway; Department of Global Public Health and Primary Care, University of Bergen, Bergen, Norway; Institute for Health Metrics and Evaluation, University of Washington, Seattle, Washington, United States. Elisabete Weiderpass, PhD, Department of Medical Epidemiology and Biostatistics, Karolinska Institutet, Stockholm, Sweden; Department of Research, Cancer Registry of Norway, Institute of Population-Based Cancer Research, Oslo, Norway; Department of Community Medicine, Faculty of Health Sciences, University of Troms $\varnothing$, The Arctic University of Norway, Tromsø, Norway; Genetic Epidemiology Group, Folkhälsan Research Center, Helsinki, Finland. Robert G. Weintraub, MBBS, Royal Children's Hospital, Melbourne, VIC, Australia; The University of Melbourne, Melbourne, VIC, Australia; Murdoch Children's Research Institute, Melbourne, VIC, Australia. Andrea Werdecker, PhD, Competence Center Mortality-Follow-Up of the German National Cohort, Federal Institute for Population Research, Wiesbaden, Germany. Harvey A. Whiteford, PhD, School of Public Health, University of Queensland, Brisbane, Queensland, Australia; Queensland Centre for Mental Health Research, Brisbane, Queensland, Australia; Institute for Health Metrics and Evaluation, 
University of Washington, Seattle, United States. Yuichiro Yano, MD, Department of Preventive Medicine, Northwestern University, Chicago, Illinois, United States. Mehdi Yaseri, PhD, Tehran University of Medical Sciences, Terhan, Iran; Ophthalmic Research Center, Shahid Beheshti University of Medical Sciences, Tehran, Iran. Naohiro Yonemoto, MPH, Department of Biostatistics, School of Public Health, Kyoto University, Kyoto, Japan. Mustafa Z. Younis, DrPH, Jackson State University, Jackson, MS, United States. Chuanhua $\mathrm{Yu}, \mathrm{PhD}$, Department of Epidemiology and Biostatistics, School of Public Health, Wuhan University, Wuhan, China; Global Health Institute, Wuhan University, Wuhan, China. Aisha O. Jumaan, $\mathrm{PhD}$, Independent Consultant, Seattle, Washington, United States. Theo Vos, PhD, Institute for Health Metrics and Evaluation, University of Washington, Seattle, Washington, United States. Simon I. Hay, DSc, Oxford Big Data Institute, Li Ka Shing Centre for Health Information and Discovery, University of Oxford, Oxford, United Kingdom; Institute for Health Metrics and Evaluation, University of Washington, Seattle, Washington, United States. Mohsen Naghavi, $\mathrm{PhD}$, Institute for Health Metrics and Evaluation, University of Washington, Seattle, Washington, United States. George C. Patton, MD, Murdoch Childrens Research Institute, Department of Paediatrics, University of Melbourne, Melbourne, Victoria, Australia. Christopher J. L. Murray, DPhil, Institute for Health Metrics and Evaluation, University of Washington, Seattle, Washington, United States.

\section{Compliance with ethical standards}

This manuscript reflects original work that has not previously been published in whole or in part and is not under consideration elsewhere. All authors have read the manuscript and have agreed that the work is ready for submission and accept responsibility for its contents. The authors of this paper have complied with all ethical standards and do not have any conflicts of interest to disclose at the time of submission. The funding source played no role in the design of the study, the analysis and interpretation of data, and the writing of the paper. The study did not involve human participants and/or animals; therefore, no informed consent was needed.

Conflict of interest The authors declare that they have no conflicts of interest at this time.

Funding statement GBD 2015 is funded by Bill \& Melinda Gates Foundation.

Open Access This article is distributed under the terms of the Creative Commons Attribution 4.0 International License (http://crea tivecommons.org/licenses/by/4.0/), which permits unrestricted use, distribution, and reproduction in any medium, provided you give appropriate credit to the original author(s) and the source, provide a link to the Creative Commons license, and indicate if changes were made.

\section{References}

Alaovie A, Ali A, Afifi R, Coalition AYH (2017) Arab youth respond to the Lancet Commission on Adolescent Health and Wellbeing. Lancet 389:1964-1965

Blair I, Grivna M, Sharif AA (2014) The "Arab World" is not a useful concept when addressing challenges to public health. Public Health Educ Res Middle East Front Public Health 2:30. doi: $10.3389 /$ fpubh. 2014.00030
Cole TJ, Lobstein T (2012) Extended international (IOTF) body mass index cut-offs for thinness, overweight and obesity. Pediatric obesity 7:284-294. doi:10.1111/j.2047-6310.2012.00064.x

Franks PW, Hanson RL, Knowler WC, Sievers ML, Bennett PH, Looker HC (2010) Childhood obesity, other cardiovascular risk factors, and premature death. N Engl J Med 362:485-493. doi:10.1056/NEJMoa0904130

GBD DALYs Hale Collaborators (2016) Global, regional, and national disability-adjusted life-years (DALYs) for 315 diseases and injuries and healthy life expectancy (HALE), 1990-2015: a systematic analysis for the Global Burden of Disease Study 2015. Lancet 388:1603-1658. doi:10.1016/S0140-6736(16)3 1460-X

GBD Disease Injury Incidence and Prevalence Collaborators (2016) Global, regional, and national incidence, prevalence, and years lived with disability for 310 diseases and injuries, 1990-2015: a systematic analysis for the global burden of disease study 2015 . Lancet 388:1545-1602. doi:10.1016/S0140-6736(16)31678-6

GBD Mortality and Causes of Death Collaborators (2016) Global, regional, and national life expectancy, all-cause mortality, and cause-specific mortality for 249 causes of death, 1980-2015: a systematic analysis for the global burden of disease study 2015 . Lancet 388:1459-1544. doi:10.1016/S0140-6736(16)31012-1

Gbd SDG Collaborators (2016) Measuring the health-related sustainable development goals in 188 countries: a baseline analysis from the global burden of disease study 2015. Lancet. doi:10. 1016/S0140-6736(16)31467-2

GBD Tobacco Collaborators (2017) Smoking prevalence and attributable disease burden in 195 countries and territories, 1990-2015: a systematic analysis from the global burden of disease study 2015. Lancet. doi:10.1016/S0140-6736(17)30819-X

GBD 2015 Eastern Mediterranean Region Cardiovascular Disease Collaborators, Mokdad AH (2017) Burden of cardiovascular diseases in the Eastern Mediterranean Region, 1990-2015: findings from the global burden of disease 2015 study. Int $\mathbf{J}$ Public Health. doi:10.1007/s00038-017-1012-3

GBD 2015 Eastern Mediterranean Region Collaborators, Mokdad AH (2017) Danger ahead: the burden of diseases, injuries, and risk factors in the Eastern Mediterranean Region, 1990-2015. Int J Public Health. doi:10.1007/s00038-017-1017-y

GBD 2015 Eastern Mediterranean Region HIV/AIDS Collaborators, Mokdad AH (2017) Trends in HIV/AIDS morbidity and mortality in Eastern Mediterranean countries, 1990-2015: findings from the global burden of disease 2015 study. Int J Public Health. doi:10.1007/s00038-017-1023-0

GBD 2015 Eastern Mediterranean Region Intentional Injuries Collaborators, Mokdad AH (2017) Intentional injuries in the Eastern Mediterranean Region, 1990-2015: findings from the global burden of disease 2015 study. Int J Public Health. doi:10. 1007/s00038-017-1005-2

GBD 2015 Eastern Mediterranean Region Mental Health Collaborators, Mokdad AH (2017) The burden of mental disorders in the Eastern Mediterranean Region, 1990-2015: findings from the global burden of disease study 2015. Int J Public Health. doi:10. 1007/s00038-017-1006-1

Mandil A, Chaaya M, Saab D (2013) Health status, epidemiological profile and prospects: Eastern Mediterranean region. Int J Epidemiol 42:616-626. doi:10.1093/ije/dyt026

Maziak W, Ward KD, Afifi Soweid RA, Eissenberg T (2004) Tobacco smoking using a waterpipe: a re-emerging strain in a global epidemic. Tob Control 13:327-333. doi:10.1136/tc.2004.008169

Mokdad AH, Jaber S, Abdel Aziz MI et al (2014) The state of health in the Arab world, 1990-2010: an analysis of the burden of diseases, injuries, and risk factors. Lancet 383:309-320. doi:10. 1016/S0140-6736(13)62189-3 
Mokdad AH, Forouzanfar MH, Dauod F et al (2016a) Health in times of uncertainty in the eastern Mediterranean region, 1990-2013: a systematic analysis for the Global Burden of Disease Study 2013. Lancet Glob Health 4:e704-e713. doi:10.1016/S2214109X(16)30168-1

Mokdad AH, Forouzanfar MH, Dauod F et al (2016b) Global burden of diseases, injuries, and risk factors for young people's health during 1990-2013: a systematic analysis for the global burden of disease study 2013. Lancet 387:2383-2401. doi:10.1016/S01406736(16)00648-6

Patton GC, Coffey C, Cappa C et al (2012) Health of the world's adolescents: a synthesis of internationally comparable data. Lancet 379:1665-1675 (S0140-6736(12)60203-7 [pii])

Patton GC, Sawyer SM, Santelli JS et al (2016) Our future: a Lancet commission on adolescent health and wellbeing. Lancet 387:2423-2478. doi:10.1016/S0140-6736(16)00579-1

Sawyer SM, Afifi RA, Bearinger LH, Blakemore SJ, Dick B, Ezeh AC, Patton GC (2012) Adolescence: a foundation for future health. Lancet 379:1630-1640. doi:10.1016/S0140-6736(12)60 $072-5$

The Global Burden of Disease Child and Adoelscent Health Collaboration (2017) Child and adolescent health from 1990 to 2015: findings from the global burden of diseases, injuries, and risk factors 2015 study. JAMA Pediatr. doi:10.1001/jamapedia trics.2017.0250

The World Bank (2006) Development and the next generation. http:// documents.worldbank.org/curated/en/556251468128407787/pdf/ 359990WDR0complete.pdf. Accessed 12 Jul 2017
Tylee A, Haller DM, Graham T, Churchill R, Sanci LA (2007) Youthfriendly primary-care services: how are we doing and what more needs to be done? Lancet 369:1565-1573 (S0140-6736(07) 60371-7 [pii])

UNICEF (2016a) Female genital mutilation/cutting: a global concern. https://www.unicef.org/media/files/FGMC_2016_brochure_ final_UNICEF_SPREAD.pdf. Accessed 12 Jul 2017

UNICEF (2016b) Good practices in adolescent and youth programming. https://goodpracticessite.files.wordpress.com/2016/03/1good-practices_compressed1.pdf. Accessed 12 Jul 2017

United Nations Inter-Agency Technical Task Team on Young People [UN IATTTYP] (2015) Regional framework of joint strategic actions for young people in the Arab States and Middle East and North Africa region. http://documents.wfp.org/stellent/groups/ public/documents/op_reports/wfp282333.pdf. Accessed $12 \mathrm{Jul}$ 2017

Viner RM, Ozer EM, Denny S, Marmot M, Resnick M, Fatusi A, Currie C (2012) Adolescence and the social determinants of health. Lancet 379:1641-1652. doi:10.1016/S0140-6736(12)60 $149-4$

WHO (2017a) Global accelerated action for the health of adolescents (AA-HA!): guidance to support country implementation. http:// apps.who.int/iris/bitstream/10665/255415/1/9789241512343eng.pdf?ua=1. Accessed 12 Jul 2017

WHO (2017b) WHO regional office for the Eastern Mediterranean. http://www.emro.who.int/entity/about-us/index.html. Accessed 12 Jul 2017 